\title{
An unexpected acute coronary syndrome
}

\author{
W G van Hougenhouck-Tulleken, Cert Nephro (SA), FCP (SA); OrcID 0000-0003-4625-0139; \\ V Ueckermann, MMed (Int Med); OrcID 0000-0002-4419-3583
}

Department of Internal Medicine, Faculty of Health Sciences, University of Pretoria and Steve Biko Academic Hospital, Pretoria, South Africa

Corresponding author: W van Hougenhouck-Tulleken (westulleken@gmail.com)

\begin{abstract}
Acute coronary syndromes due to coronary artery vasospasm occur rarely and are difficult to diagnose in the acute setting. We present a patient with hypocalcaemia-induced coronary artery vasospasm, which resulted in an acute ST-elevation myocardial infarction. This was reversible upon administering intravenous calcium, with no long-term cardiac consequences for our patient.

Keywords. Acute coronary syndrome, bridging myocardium, chronic kidney disease, hypocalcaemia, pancreatitis.

S Afr J Crit Care 2019;35(1):26-27. https://doi.org/10.7196/SAJCC.2019.v35i1.386
\end{abstract}

\begin{abstract}
Contribution of study
- Hypocalcaemia is a common clinical problem that is usually clinically quiescent, but in the acute setting can present with dramatic signs and symptoms.

- Hypocalcaemia resulting in an acute coronary syndrome is usually rapidly reversed by administering intravenous calcium.

- A hypocalcaemia-induced acute coronary syndrome is most likely a function of the myocardium being perfused primarily during diastole, with partial vasospasm limiting the diastolic flow during times of increased cardiac output or energy requirements.
\end{abstract}

\section{Case}

A 41-year-old man was admitted to the intensive care unit at Steve Biko Academic Hospital with acute pancreatitis, suspected clinically based on a raised amylase level (450 U/L; reference range: 20 - $104 \mathrm{U} / \mathrm{L})$, and later confirmed on a computed tomography (CT) scan. Pancreatitis was due to the use of herbal medication and a cytomegalovirus infection (viral load: $5472 \mathrm{U} / \mathrm{L}$ ). The patient was HIV co-infected (stage 4 according to World Health Organization staging guidelines), but highly active antiretroviral therapy had not been initiated. In addition, he had stage 3 chronic kidney disease (CKD), proven on biopsy to be due to HIVrelated focal segmental glomerulosclerosis, complicated by anaemia and secondary hyperparathyroidism. The patient developed severe, acute hypocalcaemia (corrected serum calcium level: $1.09 \mathrm{mmol} / \mathrm{L}$; reference range: $2.15-2.50 \mathrm{mmol} / \mathrm{L}$ ), precipitated by pancreatitis-induced saponification and aggressive administration of saline. Clinically, this presented as a positive Trousseau's sign during blood pressure measurement and a prolonged corrected QT (QTc) interval (360 ms) on the electrocardiograph upon admission (Fig. 1A). The patient became unexpectedly unstable 24 hours post admission and developed acute pulmonary oedema that required ventilation. An electrocardiogram performed at this time revealed ST elevation in keeping with an acute myocardial infarction (Fig. 1B). Serum troponins were initially within the normal range ( $40 \mathrm{ng} / \mathrm{L}$ ), but later became markedly elevated (peak: $4358 \mathrm{ng} / \mathrm{L}$ ). His urine output remained $>1 \mathrm{~mL} / \mathrm{kg} / \mathrm{h}$.

An acute ST-elevation myocardial infarction was diagnosed and a thrombolytic agent was prescribed, while intravenous calcium replacement had been initiated as part of his resuscitation. The patient stabilised before the thrombolytic agent could be administered, with complete resolution of the ST elevation and no residual Q waves (Fig. 1C).
The thrombolytic agent was not given subsequently. The patient made an uneventful recovery and was discharged to the ward 5 days later.

Various investigations were conducted in relation to the cardiac insult. A technetium sestamibi scan of the patient's myocardium revealed no perfusion defects (Fig. 2), whereas a non-contrast CT scan of the chest revealed no coronary artery calcification or plaques. A cardiac magnetic resolution imaging (MRI) scan revealed normal coronary arteries, with no areas of stenosis and no bridging myocardium. A coronary angiogram was contraindicated owing to the patient's CKD.

\section{Discussion}

Hypocalcaemia is commonly encountered clinically, but for the majority of patients it is indolent and clinically silent; a prolonged QT interval on the electrocardiograph is often the only clinical clue to hypocalcaemia. However, acute hypocalcaemia can present dramatically, with diaphoresis, psychiatric abnormalities, seizures, cardiac failure, bronchospasm or tetany. ${ }^{[1]}$ The pathognomonic feature of acute hypocalcaemia is tetany (typified by a Trousseau's sign) and is usually preceded by paraesthesia (peri-oral and acral), increased neuromuscular irritability (Chvostek's sign) and muscle cramps. ${ }^{[1,2]}$ Cardiac dysfunction due to hypocalcaemia is commonly seen as dysrhythmias (most frequently a prolonged QT interval and rarely torsade de pointes), but may manifest as congestive heart failure or, infrequently, as an acute coronary syndrome. ${ }^{[3-5]}$

Acute hypocalcaemia is a rare cause of coronary artery spasm, and even more so when triggered by CKD and pancreatitis. ${ }^{[3-6]}$ The concept of hypocalcaemia-induced coronary artery vasospasm syndrome is contentious, as it seems unlikely that a single artery would undergo sufficient vasospasm to cause ischaemia, without another artery of 


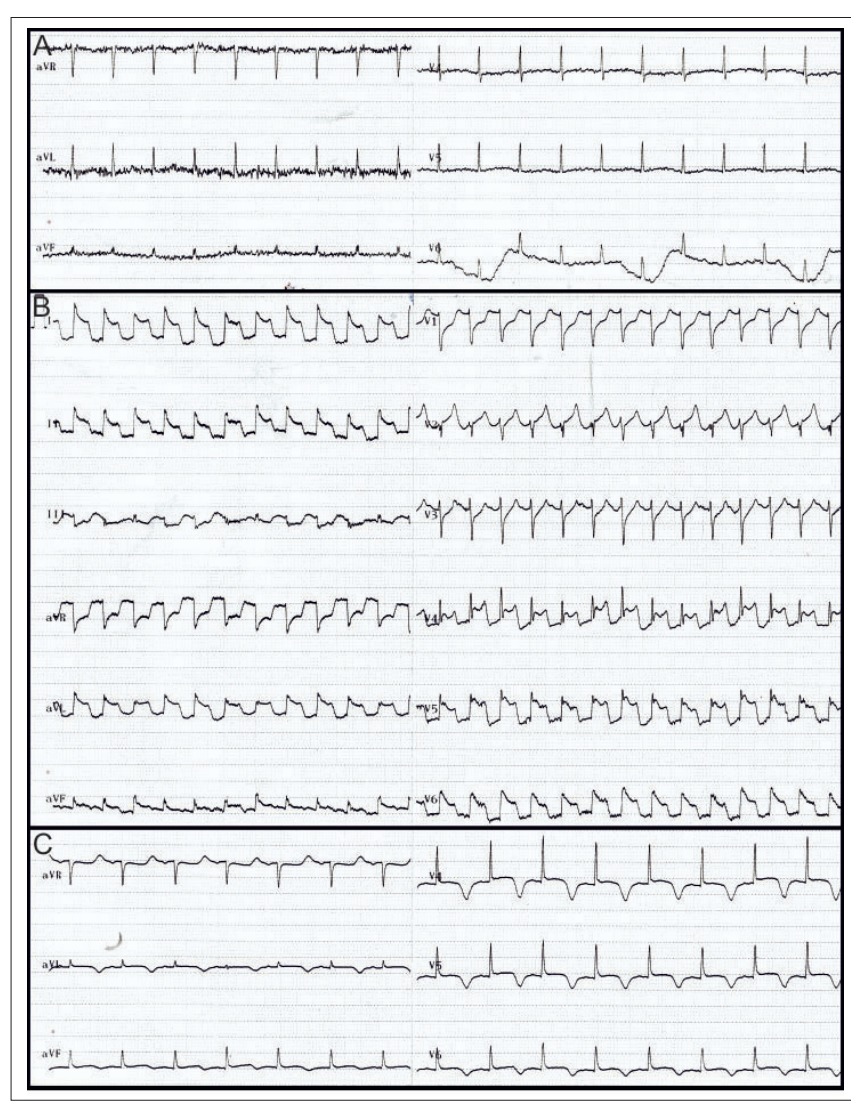

Fig. 1A. Admission ECG showing sinus tachycardia; QTc=509 ms (120 bpm, QT interval: $360 \mathrm{~ms}$ ). No ST changes are seen.

Fig. 1B. ECG at decompensation, showing sinus tachycardia; QTc=580 ms (156 bpm, QT interval: $260 \mathrm{~ms}$ ). ST elevation is seen during V4 - V6, I, II, aVL and aVF, with reciprocal changes during V1, aVR and III, in keeping with a large leftcirculation ST-elevation myocardial infarct.

Fig. 1C. ECG 15 minutes post intravenous calcium replacement. Sinus tachycardia is seen; QTc $=637 \mathrm{~ms}$ (90 bpm, QT interval: $520 \mathrm{~ms}$ ). ST elevation has resolved and no $Q$ waves are seen.

$E C G=$ electrocardiograph; $Q T c=$ corrected $Q T$ interval; $b p m=$ beats per minute.

similar calibre causing ischaemic symptoms at a distant site. The counter-argument to this is that the cardiac blood supply is unique, with the majority of the myocardium being perfused during diastole. ${ }^{[7]}$ Conceivably, ischaemia could result from partial coronary artery spasm due to hypocalcaemia if diastolic myocardial blood flow decreased below a critical threshold, thereby causing a supply-demand mismatch. This would be worsened by a tachycardia-induced i-crease in cardiac oxygen requirements. In our patient, we extended the concept of vasospasm and hypothesised that if he had myocardial bridging, the bridge might have become tetanic, which, even with incomplete occlusion of the coronary vessel, could have resulted in ischaemia. However, on review of his cardiac MRI scan, no myocardial bridge was found.

The decision to replace calcium first and prescribe a thrombolytic agent thereafter in our patient was serendipitous. The diagnosis of hypocalcaemia-induced coronary artery vasospasm is difficult to make

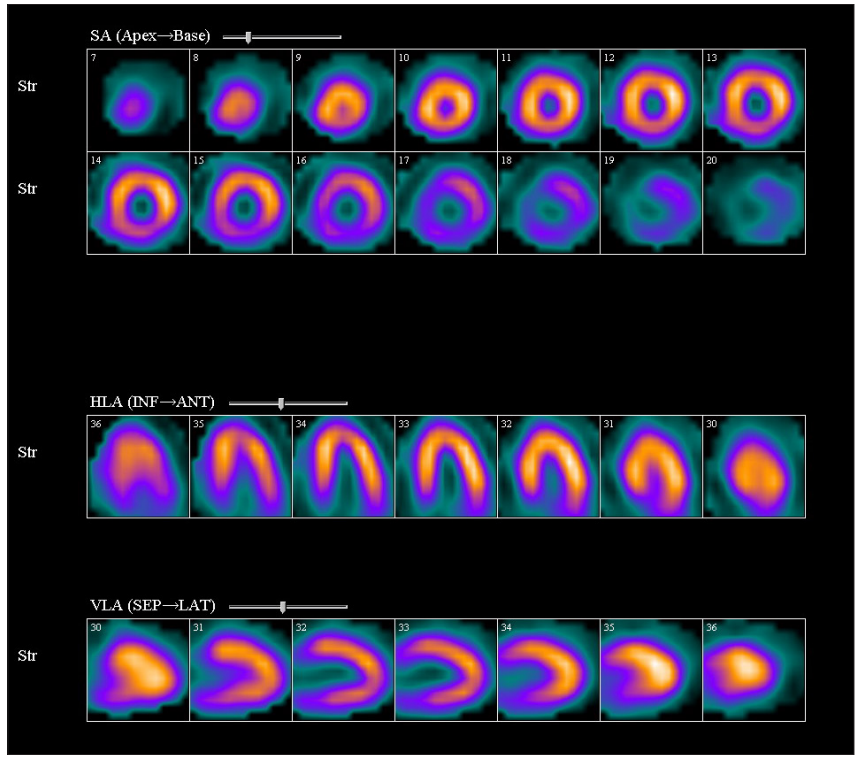

Fig. 2. Technetium (99mTc) sestamibi stress test scan, showing no perfusion defects. $S A=$ short axis; HLA = horizontal long axis; VLA = vertical long axis; $I N F=$ inferior $; A N T=$ anterior $; S E P=$ septum; LAT = lateral wall .

in the acute setting, especially in a patient with multiple risk factors such as CKD and HIV infection. To our knowledge, there are no guidelines to assist the clinician in this rare situation. However, a prudent approach in such a case could be to proceed with calcium replacement and then, if there is no immediate response, to administer the lytic in accordance with local protocols.

Acknowledgements. We thank the patient for his consent to publish the data in an anonymous manner. Ethical clearance was obtained from the University of Pretoria’s Health Research Ethics Committee (ref. no. 64/2019). Author contributions. WvH-T was responsible for manuscript preparation, while VU responsible for manuscript revision.

Funding. None.

Conflicts of interest. None.

1. Tohme JF, Bilezikian JP. Hypocalcemic emergencies. Endocrinol Metab Clin North Am 1993;22(2):363-375. https://doi.org/10.1016/s0889-8529(18)30171-3

2. Shafer AL, Shoback DM. Hypocalcemia: Diagnosis and treatment. In: Feingold KR, Anawalt B Boyce A, et al., eds. Endotext. South Dartmouth: MDText.com, 2000. https://www.ncbi.nlm.nih. gov/books/NBK279022/

3. Kozeny GA, Ragona BP, Bansal VK, et al. Myocardial infarction with normal results of coronary angiography following diltiazem withdrawal. Am J Med 1986;80(6):1184-1186. https://doi org/10.1016/0002-9343(86)90682-0

4. Gómez-Domínguez R, Hidalgo R, García-Rubira JC. Severe hypocalcemia masquerading as acute coronary syndrome. J Emerg Med 2013;45(5):715-717. https://doi.org/10.1016/j. jemermed.2013.01.047

5. Rasoul S, Dambrink JE. Recurrent acute coronary syndrome due to coronary artery spasm in different coronary arteries. J Cardiol Cases 2013;8(3):95-98. https://doi.org/10.1016/j. jccase.2013.05.007

6. Adeel MY, Clarke JD, Shetty S, Arora A, Buscher MG. Severe hypocalcemia mimicking acute inferior ST-segment elevation myocardial infarction. Oxf Med Case Reports 2018;2018(12):438441. https://doi.org/10.1093/omcr/omy103

7. Ishikawa Y, Kawawa Y, Kohda E, Shimada K, Ishii T. Significance of the anatomical properties of a myocardial bridge in coronary heart disease. Circ J 2011;75(7):1559-1566. https://doi. org/10.1253/circj.cj-10-1278

Reviewed 24 March 2019. Accepted 22 May 2019. 